\title{
Forearm Compartment Syndrome in a Two-year-old Boy with Severe Hemophilia A and High Titer Inhibitor Factor VIII: A Case Report
}

\author{
Takuya Sato $^{1,2^{*}}$, Masuo Sasa $\mathrm{MD}^{2}$, Takeshi Arai ${ }^{1}$ and Hisateru Niki ${ }^{1}$ \\ ${ }^{1}$ Department of Orthopaedic Surgery, St. Marianna University School of Medicine, Sugao Miyamaeku Kawasaki \\ Kanagawa, Japan
}

${ }^{2}$ Department of Orthopedic Surgery, St. Marianna University Yokohama Seibu hospital Orthopedics department, Yasashi Asahi-ku Yokohama, Kanagawa, Japan

"Corresponding author: Takuya Sato, Department of Orthopedic Surgery, St. Marianna University School of Medicine, 216-1 Sugao Miyamaeku Kawasaki Kanagawa 216-8511, Japan, Tel: +81449768111; Fax: +81449779683; Email: satotaku@kg8.so-net.ne.jp

\begin{abstract}
We present a case of a two-year-old boy with hemophilia $\mathrm{A}$ and high titer factor inhibitor, who developed forearm compartment syndrome two days after taking blood samples for a regular check-up of serum concentration of Factor VIII. Emergency fasciotomy was performed to prevent Volkmann contracture. The patient needed bypass therapies including recombitant activated factor VII (rFVIIa), activated prothrombin complexes (aPCC), factors VII • X (MC710), and transfusions. Eleven days after the surgical intervention, he was discharged without any functional loss in the affected upper extremity. Early surgical intervention prevented compartment syndrome of affected upper extreme.
\end{abstract}

Keywords: Hemophilia; Inhibitor; Compartment syndrome; Fasciotomy

Received Date: June 06, 2018; Accepted Date: June 30, 2018; Published Date: July 7, 2018

\section{Introduction}

Compartment syndrome as well as hemorrhage and congestion associated with improper plaster casting may cause Volkmann contracture. Compartment syndrome leads to intramuscular pressure, which increases compartment space and causes permanent muscle and nerve damage, limb loss, rhabdomyolysis, and severe functional loss. With a prompt, accurate diagnosis, the treatment is emergency fasciotomy to the affected area. In general, hemophilia patients need to replenish the insufficient factor on a regular basis. In addition, 20\%-30\% of hemophilia patients develop an inhibitor to the replaced missing factor. Hemophilia patients with a high titer inhibitor need to have bypass therapy, which replenishes the other coagulation factors, inducing the factor indirectly. In these cases, it is more difficult to control the bleeding.

\section{Case Presentation}

A two-year-old boy visited the pediatric department with extreme pain in the left upper extremity and bruising of the arm.

Citation: Takuya Sato, Forearm Compartment Syndrome in a Two-year-old Boy with Severe Hemophilia A and High Titer Inhibitor Factor VIII: A Case Report. J Clin Cases Rep 1(2): 90-95. DOI: https://doi.org/10.46619/joccr.2018.1-1018 
www.tridhascholars.org | July-2018

There was no history of hemophilia A in his family. At one year and two months of the age, the patient's medical history included hemorrhage over the hip area. At that time, he was diagnosed with severe hemophilia A with a factor VIII activity less than $1 \%$ compared to the control level. To treat his hemophilia at the age of one year and ten months old, the therapy was started. When he was two years old, he had a hemorrhagic episode in his left gastrocnemius muscle, developed a factor VII inhibitor, and received rFVIIa therapy to control his bleeding. After the episode, he received an intravenous port. He was injected with rFVIIa through his intravenous port. He developed a compression above the location where the blood samples were drawn for a regular check-up of concentration of $\gamma$ factor. He was permitted to go home after bleeding at the blood sample sites had been controlled. However, his mother noticed tenderness to his forearms while giving him a bath three hours after the blood samples were drawn. The next morning he woke up with discomfort in his arms. The bruise of arms had worsened, and he was hospitalized for treatment.

At the time of admission, he was active but had discomfort in his upper extremity from the left elbow to the palmar side of the wrist. Broad subcutaneous hemorrhages were observed around both elbows in the center of the blood-sampled sites.

Laboratory findings on admission included the following: WBC 11700/ $\mu$, RBC $405 \times 1000 / \mu 1$, hemoglobin $10.7 \mathrm{~g} / \mathrm{dl}$, TP 6.6 g/dl, ALB 4.4 g/dl, T-bil 0.7 mg/dl, AST 28 IU/l, ALT 10 IU/l, LDH 237 IU/l, Cr 0.2 mg/dl, BUN 11.1 mg/dl, Na 136 mEq/l, K $3.4 \mathrm{mEq} / 1$, Cl $104 \mathrm{mEq} / \mathrm{l}$, CRP less $0.3 \mathrm{mg} / \mathrm{dl}$, PT-INR 0.81, APTT $138 \mathrm{sec}, \gamma$ factor less 1\%, $\gamma$ factor inhibitor $252 \mathrm{BU} / \mathrm{ml}$. After hospitalization, the patient received injections of rFVIIa. His arms were also placed in a cool-down position to help control the swelling. However, two days after hospitalization, bruising had spread to the left palmer elbow side along with subcutaneous hemorrhage and blisters with pain (Figure 1). We determined that the patient needed an emergency fasciotomy to the affected area.

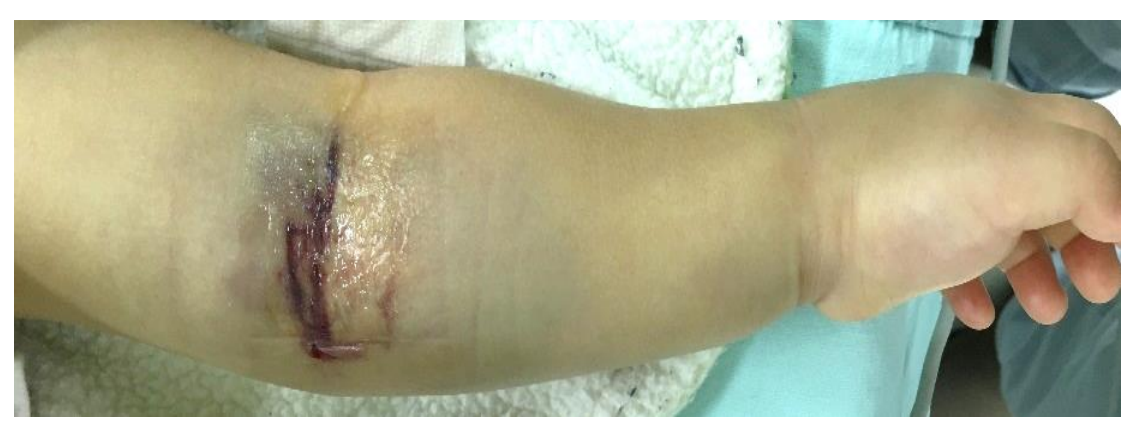

Figure 1: Preoperative finding of the affected side. Palpation, subcutaneous hemorrhage, and blisters were seen.

The surgery was performed starting at the incision, and the cut was made in S sharp fashion from elbow to the distal forearm palmer side. The subcutaneous tissue contained a hematoma. The incision was extended to release the biceps tendon membrane, then to reach the biceps tendon insertion. The area with hemorrhage and hematoma were explored. The incision was performed to cut down to the biceps tendon fascia distally to the flexor digitorum superficialis arch. We released it and made sure there was no tension to the lower layer of the humeral artery and the median nerve. Furthermore, the incision was extended distally until no congestive tissue appeared (Figure 2). After removal of the hematoma, we made sure that no active bleeding was seen. We placed a penrose drain in the wound and closed the wound with nylon suture (Figure 3 ). The right forearm was also swollen, but there were no blisters or indications of pain. A subsequent routine checkup revealed no signs of the development of compartment syndrome in the right forearm (Figure 4). 


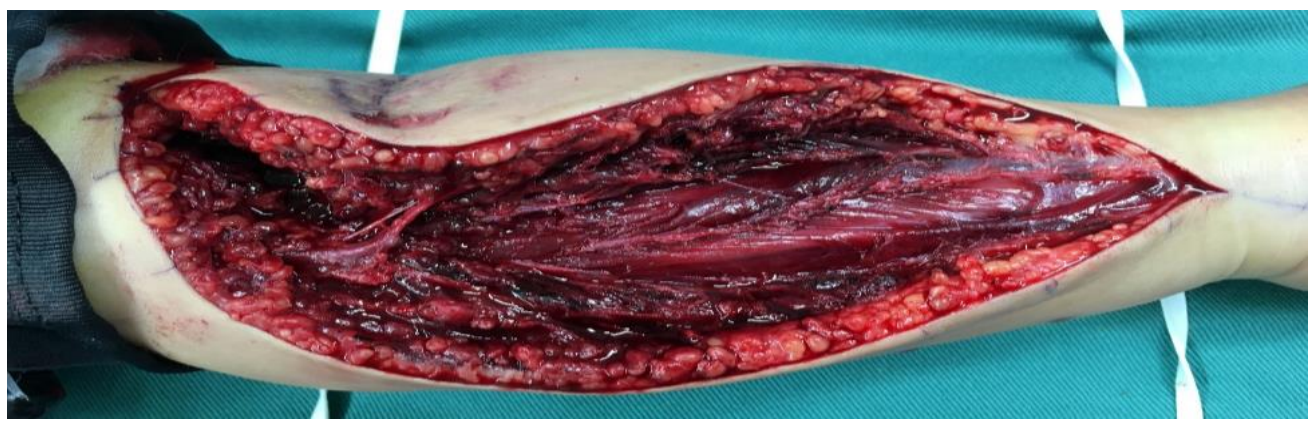

Figure 2: In operative finding of the affected forearm and hemorrhage under muscle tissue. The incision was extended until the intact tissues were seen.

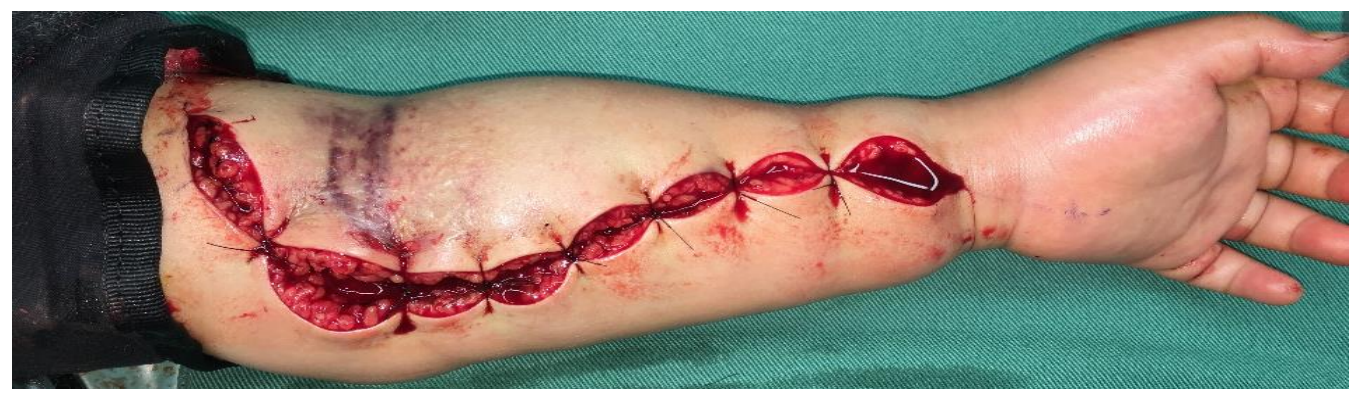

Figure 3: Forearm finding post operation and the wound was swollen with blood oozing.

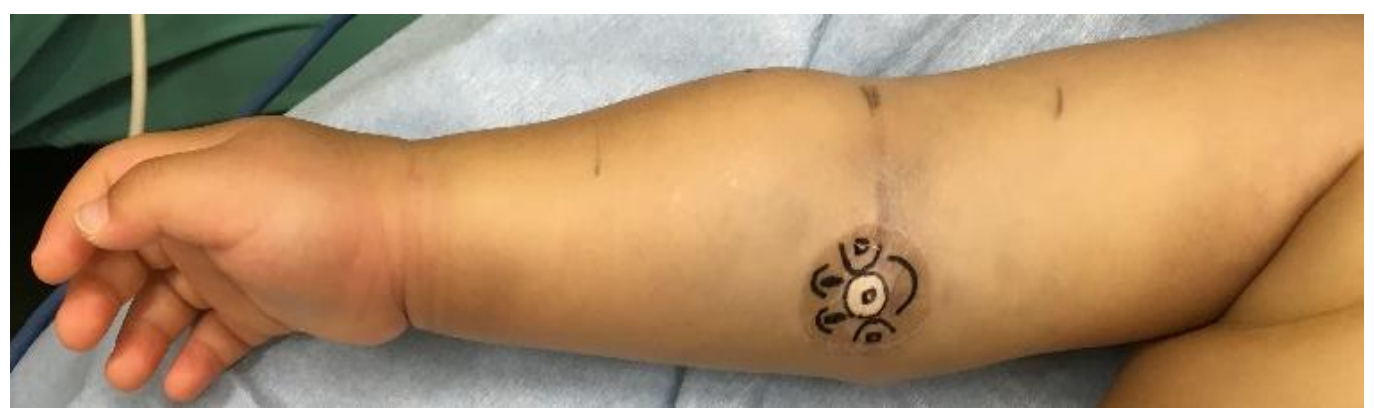

Figure 4: Forearm finding of the other side and subcutaneous hemorrhage but no blister around elbow.

After the surgery, postoperative bleeding could not be controlled with additional bypass therapies including rFV11a, aPCC, and MC7·10 formulations. The hemoglobin level was down to $6 \mathrm{~g} / \mathrm{dl}$ four days post-operation. Due to this situation, the level of factor VII was checked and replenished on frequent follow-up blood samplings.

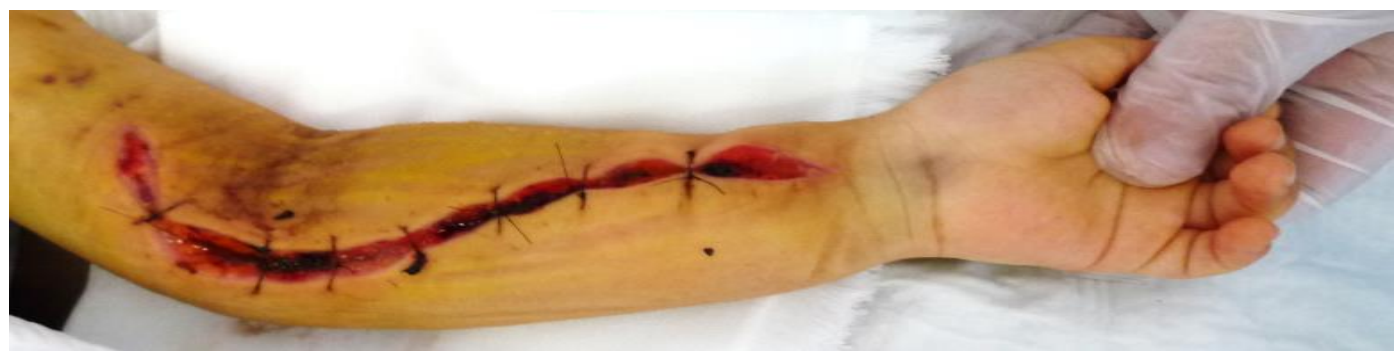

Figure 5: Forearm finding 11days after fasciotomy and the palpation was decreased and the bleeding was finally controlled. 
www.tridhascholars.org | July-2018

The total transfused products included 11 units of red cell concentrate, two units of albumin preparation, two units of fresh frozen plasma, and two units of human platelet preparation until the bleeding was finally controlled at eleven days postoperation (Figure 5 and Figure 6). The patient was discharged after 23 days without any functional loss of the upper limb (Figure 7).

\section{The course of treatment}

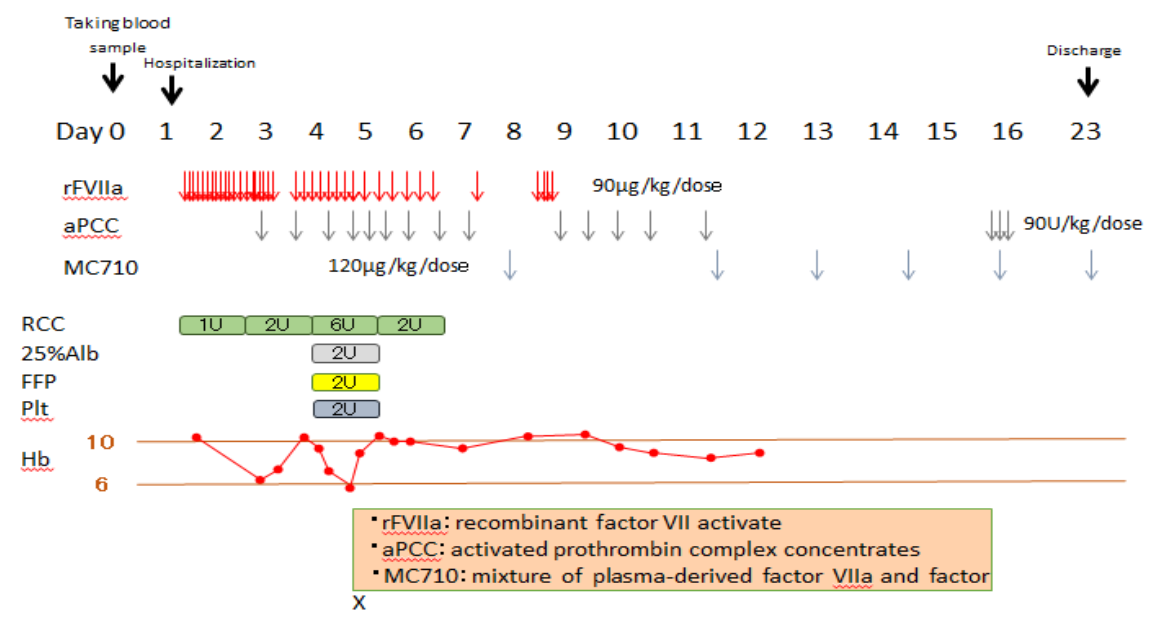

Figure 6: The course of treatment.

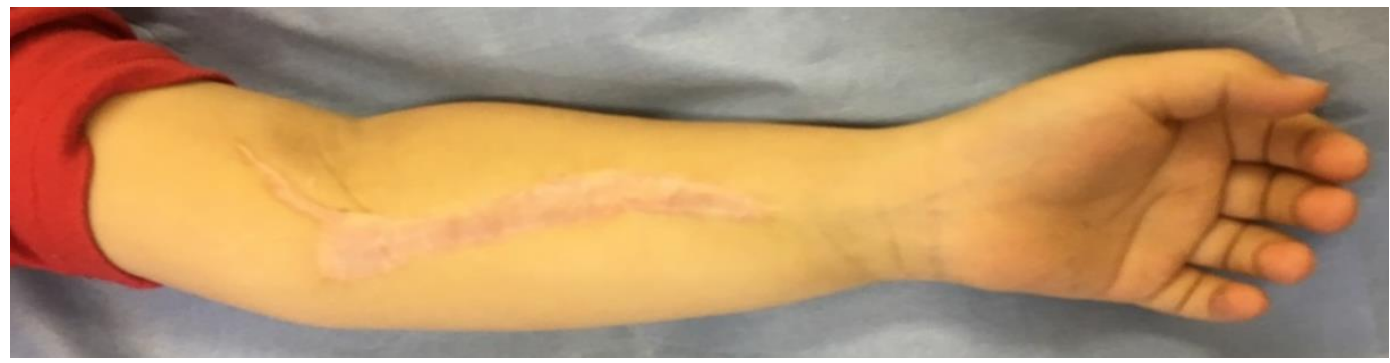

Figure 7: Finding at the follow-up and the wound is healed, no function loss of the affected side.

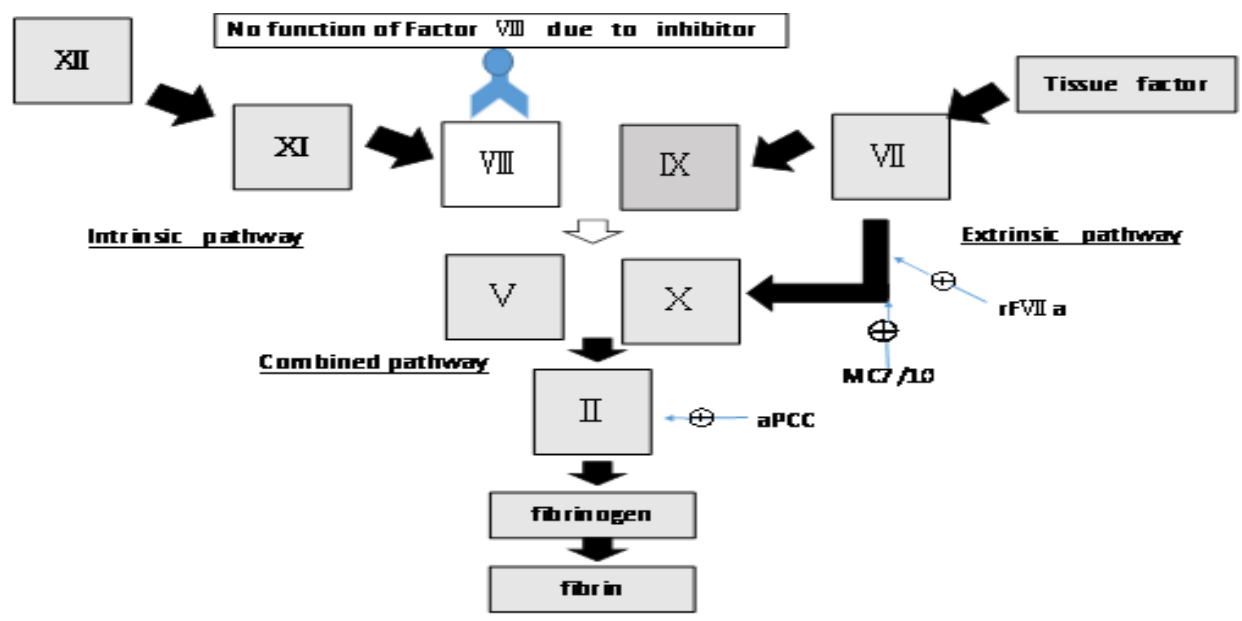

Figure 8: The schema of the bypass therapy in hemophilia VII with inhibitor. 
www.tridhascholars.org | July-2018

\section{Discussion}

In cases of severe hemophilia A with high titer inhibitor, the therapy is not the same as the factor replacement therapy in hemophilia cases without an inhibitor. The inhibitor works on the coagulation factor, deactivating its work so that coagulation becomes difficult. There are two treatments to control bleeding for hemophilia patients with an inhibitor: the first is bypass therapy; the second is inhibitor neutralization therapy, which uses high dose factor administration. The bypass therapy works on the extrinsic and combined coagulation pathway (Figure 8). The bypass therapy uses aPCC, $\mathrm{rF} \phi \mathrm{a}$, and factors $\phi \cdot \imath(M C 7 \cdot 10)$ administration. In the case of children, therapy should be administered with extra caution to prevent thrombophilia and disseminated intravascular coagulation. Tanaka et al. [1] asserts that this therapy is used worldwide according to hemophilia therapy guidelines. Hemophilia patients with a factor inhibitor are categorized into two groups. Patients who receive frequent factor VIII administration and have less than 5 Bethesda Units (BU)/ml of inhibitor are defined as low responders. High responder patients, on the other hand, have over $5 \mathrm{BU} / \mathrm{ml}$ of inhibitor. According to hemophilia therapy guidelines in Japan, first-line treatment for a low responder is inhibitor neutralization therapy, and first-line treatment for a high responder is bypass therapy.

Compartment syndrome was first described by Richard von Volkmann in 1881 [2]. This syndrome can be caused by trauma, casting, and hemorrhage. The pathophysiology of the disorder is due to a pressure increase within a confined intramuscular compartment space. This can ultimately lead to permanent muscle and nerve damage, limb loss, rhabdomyolysis, and severe functional loss. Hemophilia patients have a high risk of joint hemorrhage, but hemophilia patients who require fasciotomy are relatively rare. If a hemophilia patient with an inhibitor has hemorrhage, he needs a proper diagnosis of this condition, followed by a cooling down position of the affected site, and neutralization therapy or bypass therapy. Fasciotomy should ideally occur within six hours of a compartment syndrome diagnosis. This is supported by Kim et al. [3] who maintain that if a hemophilia patient is diagnosed with compartment syndrome, he needs to undergo fasciotomy within six hours to prevent function loss in the affected area. Moreover, Rorabeck et al. [4] found that if fasciotomy was performed within six hours, there was almost complete recovery of limb function. However, when fasciotomy was performed within 12 hours, normal limb function was regained in only $68 \%$ of patients; when performed after 24 hours, only $8 \%$ of patients regained normal function. If surgical intervention is deemed necessary, it might not only save a limb but also the patient's life. There are few reported cases [5-9] involving patients who needed fasciotomy, but of these cases, the patients had uncontrollable bleeding from the operative site during the postoperative period. In comparing adults to children who underwent fasciotomy, children required more factor administration doses because the factor activation for children is generally shorter than in adults. This is why there is more difficulty in controlling post-operative bleeding in children. In this particular case, the patient received rFVIIa as well as aPCC in bypass therapy before fasciotomy. However, the postoperative bleeding was still difficult to control even though the patient received bypass therapies as well as transfusions. The Authors who have reported on hemophilia cases with fasciotomy remark that it takes time to control the bleeding post-operation, as was true in this case. Hemophilia patients who receive fasciotomy, particularly in the case of children, require special care during hospitalization so that postoperative bleeding can be managed and controlled. 


\section{Reference}

1. Tanaka I and Midori S (2008) Current status of hemostatic treatment for patients with hemophilia and inhibitors in overseas guidelines: towards the establishment of a domestic guideline: towards the establishment of a domestic guideline. The Japanese Journal of Pediatrics Hematology 22(3): 179-187.

2. Volkmann R (1881) Ischaemic Muscle Paralyses and Contractures. Journal of Hand Surgery 801-803.

3. Kim J, Zelken J and Sacks JM (2013) Spontaneous forearm compartment syndrome in a boy with hemophilia A: a therapeutic dilemma. Eplasty: Open Access Journal of Plastic Surgery 13: 125-128.

4. Rorabeck CH and Macnab I (1975) The pathophysiology of the anterior tibial compartmental syndrome. Clinical Orthopaedics and Related Research 113: 52-57.

5. Jentzsch T, Brand-Staufer B, Schäfer FP, et al. (2014) Illustrated operative management of spontaneous bleeding and compartment syndrome of the lower extremity in a patient with acquired hemophilia A: a case report. Journal of Medical Case Reports 8 (132): 1-7.

6. Watts RG (2005) Successful use of recombinant factor VIIa for emergency fasciotomy in a patient with hemophilia A and high-titer inhibitor unresponsive to factor VIII inhibitor bypassing activity. American Journal of Hematology 79(1): 58-60.

7. Kaizuka R and Sakai M (1999) Compartment syndrome with fasciotomy in a hemophilia A and inhibitor: A case report. Japanese Journal of Pediatrics 52: 1765-1768.

8. Madigan RR, Hanna WT and Wallace SL (1981) Acute compartment syndrome in hemophilia. A case report. Journal of Bone Joint Surgery 63(8): 1327-1329.

9. Ilyas AM, Wisbeck JM, Shaffer GW et al. (2005) Upper extremity compartment syndrome secondary to acquired factor VIII inhibitor: a case report. Journal of Bone Joint Surgery 87(7): 1606-1608. 